\title{
- Dextran-Coated Magnetic Supports Modified with a Biomimetic Ligand for IgG Purification
}

\author{
3 Sara D. F. Santana, Vijaykumar L. Dhadge, and Ana C.A. Roque* \\ 4 REQUIMTE, Departamento de Química, Faculdade de Ciências e Tecnologia, Universidade Nova de Lisboa, 2829-516 Caparica, \\ 5 Portugal
}

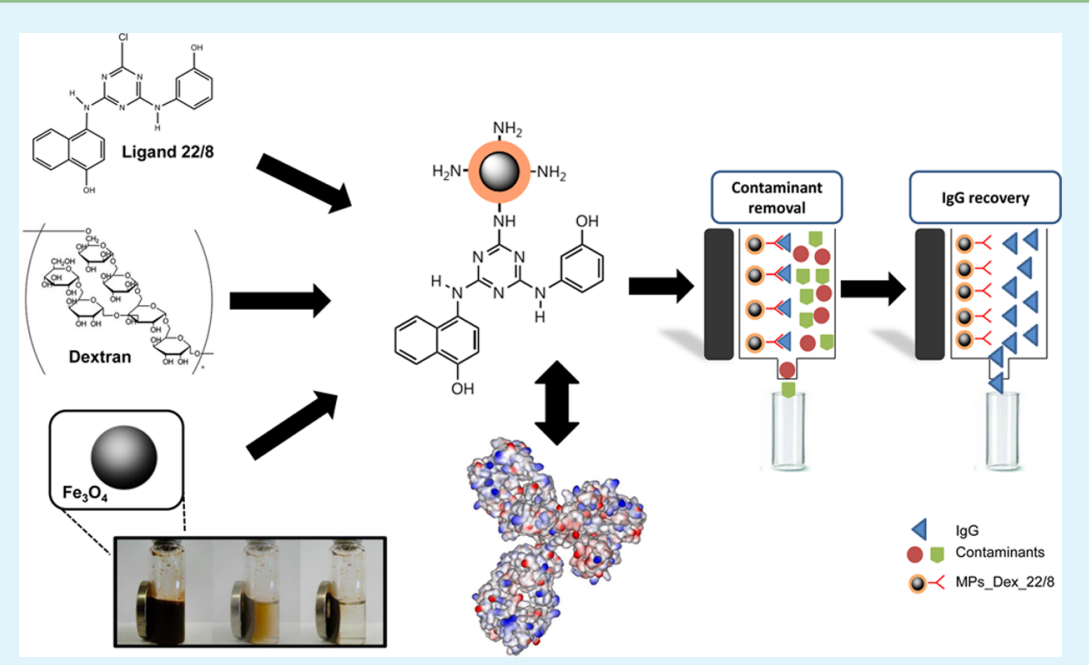

6 ABSTRACT: Dextran-coated iron oxide magnetic particles modified with ligand 22/8, a protein A mimetic ligand, were 7 prepared and assessed for IgG purification. Dextran was chosen as the agent to modify the surface of magnetic particles by 8 presenting a negligible level of nonspecific adsorption. For the functionalization of the particles with the affinity ligand toward 9 antibodies, three methods have been explored. The optimum coupling method yielded a theoretical maximum capacity for 10 human IgG calculated as $568 \pm 33 \mathrm{mg} / \mathrm{g}$ and a binding affinity constant of $7.7 \times 10^{4} \mathrm{M}^{-1}$. Regeneration, recycle and reuse of 11 particles was also highly successful for five cycles with minor loss of capacity. Moreover, this support presented specificity and 12 effectiveness for IgG adsorption and elution at $\mathrm{pH} 11$ directly from crude extracts with a final purity of $95 \%$ in the eluted fraction.

13 KEYWORDS: magnetic particles, dextran, immobilization, synthetic affinity ligand, IgG purification

\section{INTRODUCTION}

14 Full antibodies and engineered antibody formats can be 15 designed to bind to a diversity of antigens with high specificity, 16 and further conjugated with other therapeutics for increased 17 efficiency. ${ }^{1}$ For the in vivo administration of antibodies, 18 demanding production and purification processes are required 19 in order to avoid contaminations and produce safe, pure, and 20 consistent products. Simultaneously, industries have the 21 challenge to reduce total manufacturing costs. Downstream 22 processing can account for $50-80 \%$ of the total production 23 costs; therefore, there is the need to design purification 24 strategies that will target high purity and product yield as well 25 as cost minimization. ${ }^{2,3}$

26 Affinity-based methodologies are widely employed on 27 traditional antibody purification processes, and are based on 28 the selective recognition between the antibody molecule and a 29 complementary ligand immobilized in a solid matrix, commonly 30 agarose or derivatives. ${ }^{3}$ Nonspecific interactions are reduced 31 with increased yield and contaminants can be eliminated in a 32 single step. The affinity ligands mostly used to capture antibodies are biospecific ligands which are natural immuno- 33 globulin binding ligands (protein A, protein L). ${ }^{4,56}$ However, 34 these ligands are costly, labile, and can leach under certain 35 conditions. An alternative and promising choice is the use of 36 synthetic affinity ligands mimicking the biological receptors. ${ }^{7-9} 37$ Although presenting lower binding constants, the purity 38 obtained with the biomimetic ligands is still high with the 39 advantages of being inexpensive, scalable to produce, durable 40 and extraordinarily stable under harsh conditions. ${ }^{3}$ A good 41 example of biomimetic ligands toward antibodies is ligand 22/8, 42 a protein A mimetic. ${ }^{10}$ In addition, the support for ligand 43 attachment is also a key step for binding the target molecule. 44 The immobilization of ligands on agarose beads has been 45 extensively studied on literature. ${ }^{3,7}$ However, packed bed 46 chromatography and bed expanded systems present some 47 limitations, namely clogging and diffusion limitations. ${ }^{3,11} 48$

Received: August 3, 2012

Accepted: October 25, 2012 


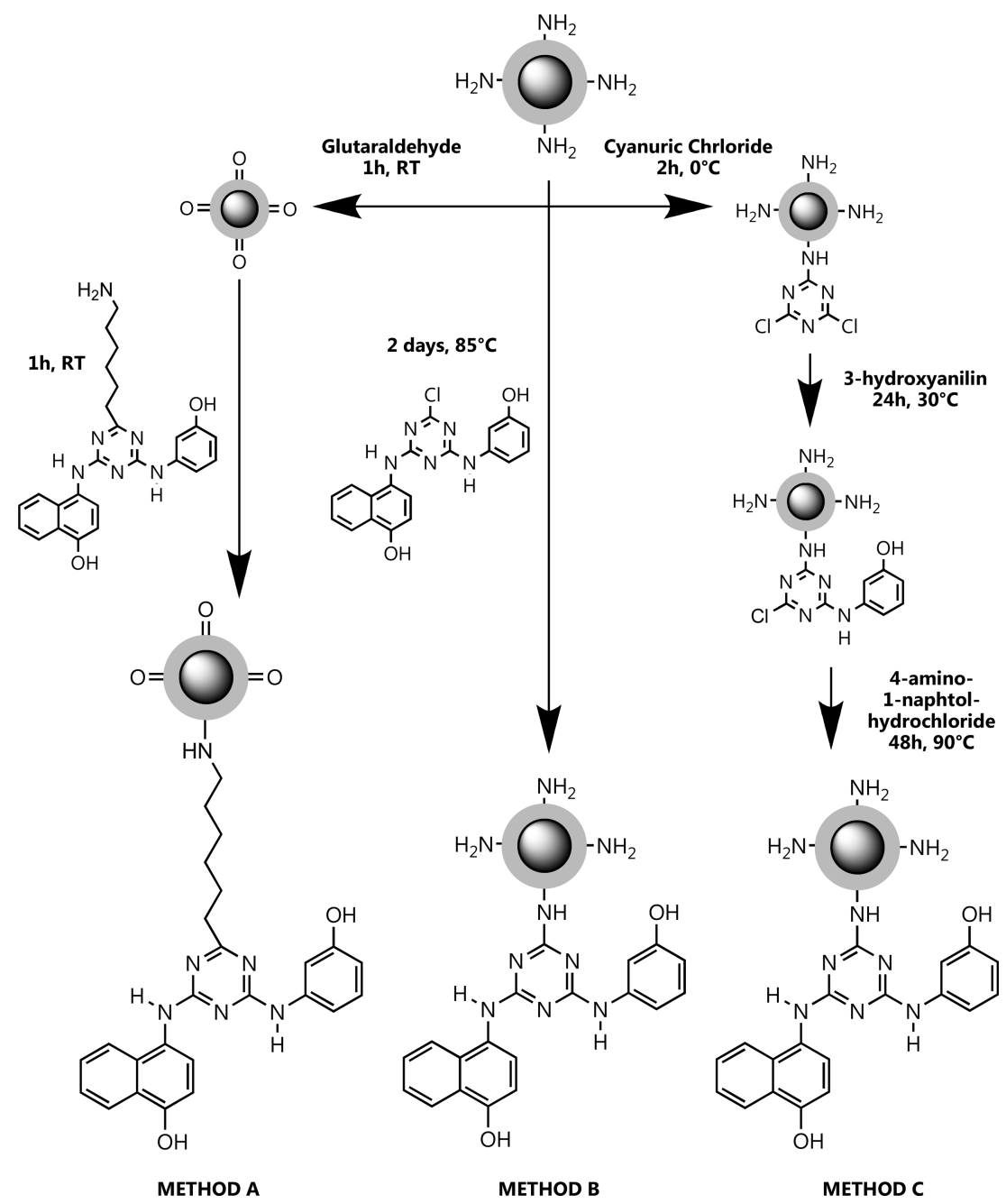

Figure 1. Schematic representation of the synthetic affinity ligand 22/8 Immobilized onto MPs coated with dextran by three different methods: method A, the ligand $22 / 8$ was used in solution phase with a six carbon spacer; method B, the ligand $22 / 8$ was also used in solution phase but without spacer; and method $\mathrm{C}$, the ligand $22 / 8$ was directly synthesized onto the support (ChemDraw 11).

49 Iron oxide magnetic particles (MPs) appear as a challenging 50 and a suitable choice for bioseparation applications because this 51 support can contribute to cost reduction and process 52 integration. ${ }^{2,3} \mathrm{MPs}$ present attractive features such as super53 paramagnetism, which greatly facilitates manipulation, recovery, 54 and reutilization, particularly in high-gradient magnetic 55 separation devices. ${ }^{12,13}$ Other advantageous characteristics of $56 \mathrm{MPs}$ concern the small size of the particles providing a high 57 surface area to volume and minimum diffusion limitations. ${ }^{14,15}$ 58 MPs present low colloidal stability because of the highly active 59 surface and high surface area to volume ratio, which increases 60 the particles agglomeration. Both phenomena have impact on 61 the size, shape, and stability of the particles. In solution, the 62 impact of these might bring some disadvantages in the 63 applicability of these supports. ${ }^{15,16}$ The coating of MPs appears 64 as an essential strategy for particle stabilization, and different 65 coating agents can be applied. MPs coating with polymers, 66 particularly biopolymers such as polysaccharides, attracted 67 attention of researchers as these are known to increase 68 biocompatibility, chemical functionality, and colloidal stability 69 of different materials. In addition, biopolymers are renewable, 70 nontoxic and biodegradable which make them an environ71 mental and sustainable choice. ${ }^{15}$ Some of the polysaccharides 72 most used for covering MPs, include agarose, ${ }^{17}$ chitosan, ${ }^{18}$ starch, ${ }^{19}$ dextran, ${ }^{20}$ and gum Arabic. ${ }^{21,22}$ Dextran, a neutral 73 polysaccharide produced by lactic acid bacteria, is a conven- 74 tional polymer used for coating MPs. MPs coated with dextran 75 (MPs_Dex) are mostly used in biomedical applications for 76 resonance magnetic imaging and there are already preparations 77 available in the market. ${ }^{15}$ These supports were also explored for 78 bioseparation and biosensing applications. ${ }^{23,24}$ In the biosepa- 79 ration field, dextran-coated MPs have already been applied for 80 the separation of proteins, ${ }^{25,26}$ cells, ${ }^{27}$ organelles, ${ }^{28}$ and for 81 isolation of target bacteria by immunomagnetic particles, ${ }^{29} 82$ through the exploitation of the natural interactions between 83 sugars and biological receptors.

This work focused on the preparation of a new magnetic 85 support, based on iron oxide magnetic particles coated with 86 dextran for bioseparation processes, taking into account the 87 characteristics of iron oxide magnetic particles coated with gum 88 Arabic (MPs_GA) previously studied. ${ }^{21}$ The novelty of this 89 work relies on the combination of a low cost and inert polymer 90 with a robust synthetic ligand mimicking protein A for the 91 purification of IgG from purified and unpurified mixtures. 92

\section{EXPERIMENTAL PROCEDURE}

Materials. (3-Aminopropyl)triethoxysilane (APTES) 98\%, 3- 93 hydroxyanilin 98\%, 4-amino-1-naphtol hydrochloride 90\%, cyanuric 94 
95 chloride $99 \%$ were acquired from Aldrich (Sintra, Portugal). Sodium 96 hydroxide $99 \%$ was purchased from Panreac (Cascais, Portugal). 97 Albumin from bovine serum, dextran from Leuconostoc mesenteroides, 98 glutaric dialdehyde $50 \mathrm{wt} \%$ sol in water, gum arabic from acacia tree, 99 iron(III) chloride hexahydrate $98 \%$, iron(II) chloride tetrahydrate 100 99\%, and N,N-dimethylformamide 99\% were acquired from Sigma 101 (Sintra, Portugal). Anthrone 97\%, sodium bicarbonate 98\%, and 102 sulfuric acid $98 \%$ were from Sigma-Aldrich (Sintra, Portugal). Human 103 normal immunoglobulin (Gammanorm) was purchased from 104 Octapharma (Lisboa, Portugal). Protein quantification assay used 105 was bichinchoninic acid (BCA) kit from Sigma. For SDS-PAGE gels, 106 the reagents used were $30 \%$ acrylamide/bis solution 37.5:1, sodium 107 dodecyl sulfate solution $10 \%$ purchased from BIO-RAD. Ammonium 108 persulphate $98 \%$ (PSA), N,N,N,N-tetramethylethylenediamine $99 \%$ 109 (TEMED), and bromphenol blue sodium salt were acquired from 110 Roth (BetaLab, Queluz, Portugal). Glycerol 99\% purchased from 111 Sigma-Aldrich (Sintra, Portugal). SDS micropellets 99\% (sodium 112 dodecyl sulfate), tris base $99.9 \%$ ultrapure for molecular biology, and 113 glycine $99 \%$ ultrapure for molecular biology were purchased from 114 NZYTech (Lisboa, Portugal). 2-Mercaptoethanol 99\% purchased from 115 Aldrich (Sintra, Portugal). Hydrochloric acid 37\% (concentrated) was 116 acquired from Panreac (Cascais, Portugal). To stain polyacrylamide 117 gels, we used the Silver Stain Plus kit from BIO-RAD (Amadora, 118 Portugal). LMW-SDS Marker Kit (18.5 kDa $-96 \mathrm{KDa})$ was from 119 NZYTech (Lisboa, Portugal).

120 Methods. Synthesis, Amination, Stability Study, and Character121 ization of Bare and Dextran-Coated MPs. Bare MPs and dextran122 coated MPs were synthesized by the coprecipitation of $\mathrm{FeCl}_{3}$ and $\mathrm{FeC}_{2}$ 123 salts, using a $\mathrm{Fe}^{2+} / \mathrm{Fe}^{3+}$ molar ratio of 0.5 , through the addition of a 124 base under an inert atmosphere, following the Massart method. ${ }^{30}$ The 125 syntheses were performed at room temperature for the bare MPs and 126 at $60{ }^{\circ} \mathrm{C}$ for the dextran-coated MPs (MPs_Dex). For the MPs_Dex, $1272.0 \mathrm{~g}$ of a $50 \mathrm{mg} / \mathrm{mL}$ aqueous solution of the biopolymer was added 128 dropwise immediately after the addition of the iron solution. The 129 synthesized MPs were washed several times with distilled water using a 130 magnet for separation. To quantify the yield of biopolymer coating 131 MPs, we analyzed the amount of biopolymer in the washes after 132 synthesisby the anthrone method. ${ }^{31}$ MPs were then aminated by using 133 3-aminopropyltriethoxy silane (APTES), ${ }^{21}$ yielding amination den134 sities of $214 \pm 44 \mu \mathrm{moL} \mathrm{NH} \mathrm{NH}_{2} / \mathrm{g}$ MPs. Finally, to evaluate the storage 135 stability at $4{ }^{\circ} \mathrm{C}$ and the stability of the supports on amination, we 136 analyzed all the washes performed in the intermediate steps by the 137 anthrone method to determine the quantity of biopolymer released. 138 All samples were characterized by Fourier transform infrared (FTIR) 139 spectroscopy on a Perkin-Elmer Spectrum BX instrument. Samples 140 were prepared by grounding and mixing with $\mathrm{KBr}$ in a proportion of 141 1:100. The magnetization of the magnetic particles in solution were 142 characterized by using a vibrating sample magnetometer (VSM) 143 (DSM 880 VSM) at INESC-MN facilities (Lisbon, Portugal). The 144 samples were prepared in milli-Q water with a concentration of 6.1 $145 \mathrm{mg} / \mathrm{mL}$ and were used $30 \mu \mathrm{L}$ of each sample in a vertical quartz rod. 146 Transmission electron microscopy (TEM) was utilized for the 147 characterization of particle morphology and estimation of the size of 148 the magnetic core. The dried particle samples were prepared by 149 evaporating dilute suspensions on a carbon-coated film and TEM 150 performed in an Analytical TEM Hitachi 8100 with Rontec standard 151 EDS detector and digital image acquisition. For all supports the 152 physical properties (hydrodynamic diameters and zeta potential) were 153 determined by Dynamic light scattering (DLS), using a Zetasizer Nano $154 \mathrm{ZS}$ from Malvern. For these analyses, samples with a final 155 concentration of $0.05 \mathrm{mg} / \mathrm{mL}$ in milli-Q water were prepared.

156 Immobilization of the Biomimetic Ligand 22/8 onto Dextran157 Coated MPs. For the immobilization of the biomimetic ligand $22 / 8$ 158 onto MPs_Dex, three different methods were tested (Figure 1). In 159 method A, the ligand $22 / 8$ has a six carbon space arm and was 160 previously synthesized in solution phase and purified ${ }^{7}$ by Dr. Abid 161 Hussain from our group. For the immobilization procedure, the 162 aminated particles $(10 \mathrm{mg} / \mathrm{mL})$ were washed five times with distilled 163 water and resuspended in a solution of glutaraldehyde with a final 164 concentration of $5 \%(\mathrm{v} / \mathrm{v})$. The suspensions were sonicated for $10 \mathrm{~min}$ and subsequently incubated for $1 \mathrm{~h}$ at room temperature with constant 165 shaking. Afterward, the particles were washed five times with milli-Q 166 water. The support was then incubated in a 1:1 stoichiometry (taking 167 into account the number of amines available) with the ligand 22/8 168 previously dissolved in DMF: $\mathrm{H}_{2} \mathrm{O}$ (50:50) and centrifuged for 5 min 169 at $13000 \mathrm{rpm}$ to make sure the insoluble part was discarded. The 170 incubation proceeded for $1 \mathrm{~h}$ at room temperature at $300 \mathrm{rpm}$ in an 171 orbital shaker. Finally, to block the remaining functional groups, we 172 washed modified supports five times with distilled water and were 173 incubated $1 \mathrm{~h}$ at room temperature with constant shaking in the 174 presence of a solution of $100 \mathrm{mMol} / \mathrm{L}$ glycine in distilled water. 175

For method B, the ligand 22/8 was synthesized in solution phase ${ }^{32} 176$ and kindly provided by Telma Barroso from our group. For this 177 immobilization procedure the aminated MPs were incubated with 5178 mol equiv (taking into account the number of amines available) of the 179 ligand 22/8 dissolved in DMF: $\mathrm{H}_{2} \mathrm{O}(1: 12)$ and with 1 equivalent of 180 sodium bicarbonate. Incubation occurred for 2 days at $85{ }^{\circ} \mathrm{C}$ with 181 constant shaking. In methods A and B, final washes were collected in 182 order to quantify the amount of ligand bound to the particles (by 183 measurement of absorbance at $280 \mathrm{~nm}$ ). However, it was not possible 184 to quantify the exact amount of ligand bound because of the extremely 185 low solubility of the ligand.

Finally, in method C, ligand $22 / 8$ was synthesized directly on the 187 particles. The aminated support was resuspended in $50 \%(\mathrm{v} / \mathrm{v}) 188$ acetone/water and reacted with $5 \mathrm{~mol}$ equiv (according to the amount 189 of amines available) of Cyanuric chloride, dissolved in acetone, during 190 $2 \mathrm{~h}$ at $0{ }^{\circ} \mathrm{C}$ at $300 \mathrm{rpm}$. In the end of this reaction, the MPs were 191 washed one time with acetone, one time with $50 \%(\mathrm{v} / \mathrm{v})$ acetone/ 192 water and finally five times with water. The first nucleophilic 193 substitution on triazine ring was then performed by adding 2194 equivalents (relative to the amount of amines) of 3-hydroxyanilin in 195 water. This reaction proceeded for $24 \mathrm{~h}$ with stirring at $30^{\circ} \mathrm{C}$ and after 196 the reaction the particles were washed five times with water. Finally, 197 for the second nucleophilic substitution, 5 mol equiv of 4-amino-1- 198 naphtol- hydrochloride, in the presence of 5 equiv. of sodium 199 hydroxide, dissolved in $50 \%(\mathrm{v} / \mathrm{v}) \mathrm{DMF} /$ water, were added to the 200 reaction and left to incubate for $48 \mathrm{~h}$ with stirring at $90{ }^{\circ} \mathrm{C}$.

After every procedure in methods A, B, and C, the particles were 202 washed sequentially with $50 \%(\mathrm{v} / \mathrm{v}) \mathrm{DMF} /$ water, water, and finally 203 resuspended in water for storage. ${ }^{21}$

Assessment of Human IgG and Bovine Serum Albumin Binding 205 to Affinity Magnetic Supports. The MPs_Dex modified with affinity 206 ligand $22 / 8(250 \mu \mathrm{L}$ at $6.0 \mathrm{mg} / \mathrm{mL})$ were tested with a pure solution 207 of human IgG (hIgG), and with a pure solution of Bovine Serum 208 Albumin (BSA). The particles suspensions were washed with 209 regeneration buffer $(0.1 \mathrm{M} \mathrm{NaOH}$ in $30 \%(\mathrm{v} / \mathrm{v})$ isopropanol), 210 followed by deionized water to neutralize the $\mathrm{pH}$. These cycles of 211 washes were repeated two times. Then, particles were equilibrated with 212 binding buffer ( $50 \mathrm{mM}$ phosphate, $\mathrm{pH} 8$ ). After preparation of the 213 supports, $250 \mu \mathrm{L}$ of a hIgG or BSA solution in binding buffer $(1 \mathrm{mg} / 214$ $\mathrm{mL}$ ) was added to the particles and incubated for $15 \mathrm{~min}$ at room 215 temperature with constant stirring, after which the supernatants were 216 separated by magnetic separation and removed. Particles were then 217 washed five times using binding buffer $(250 \mu \mathrm{L})$ following the same 218 methodology. Bound protein was then eluted with a $50 \mathrm{mM}$ Glycine- 219 $\mathrm{NaOH}, \mathrm{pH} 11$ buffer. Reuse of the modified supports were repeated 220 five times for the binding of hIgG, where after each cycle of adsorption 221 and elution the supports were regenerated two times using 222 regeneration buffer followed by deionized water to neutralize the 223 $\mathrm{pH}$. All samples were analyzed by BCA assay (microplate reader 224 assay), in order to quantify the amount of protein bound to and eluted 225 from the supports. ${ }^{21}$ Nonmodified particles (MPs and MPs_Dex) 226 were tested at the same time and in the same conditions. To assess 227 biopolymer and iron leaching, we incubated the magnetic supports 228 separately with binding, elution, and regeneration buffers, and the 229 supernatants recovered by magnetic separation. The amount of 230 biopolymer and iron in the supernatants were quantified by the 231 anthrone $^{31}$ and magnetite ${ }^{33}$ methods, respectively. Adsorption 232 isotherms of hIgG on the magnetic supports were estimated by 233 partition equilibrium experiments. Solutions of hIgG $(0-18 \mathrm{mg} / \mathrm{mL} ; 234$ 
A)

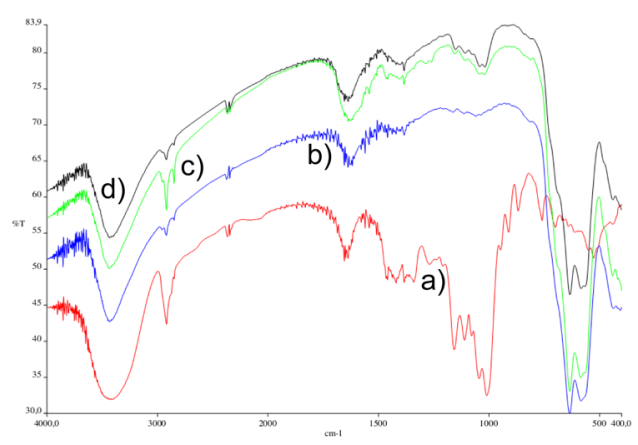

C)

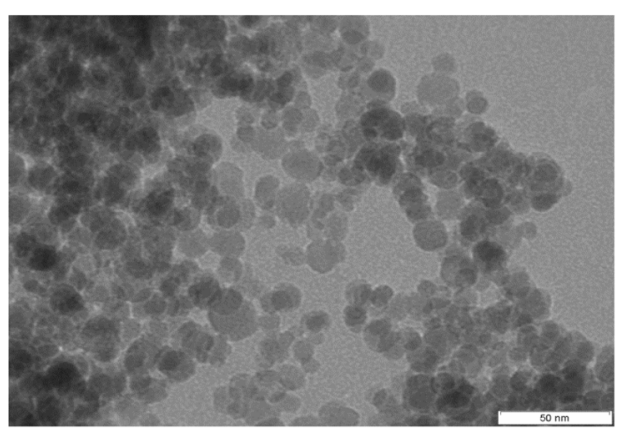

E)

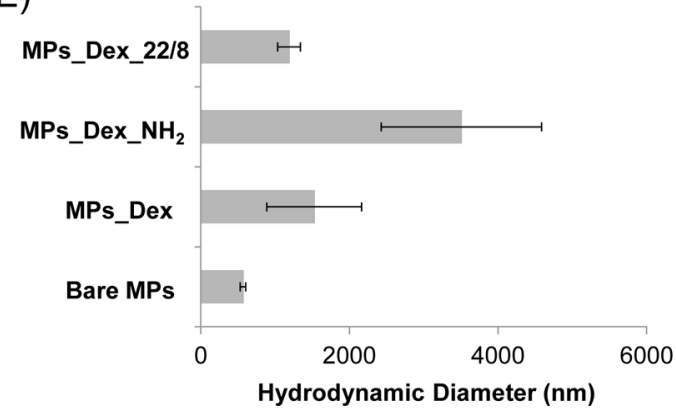

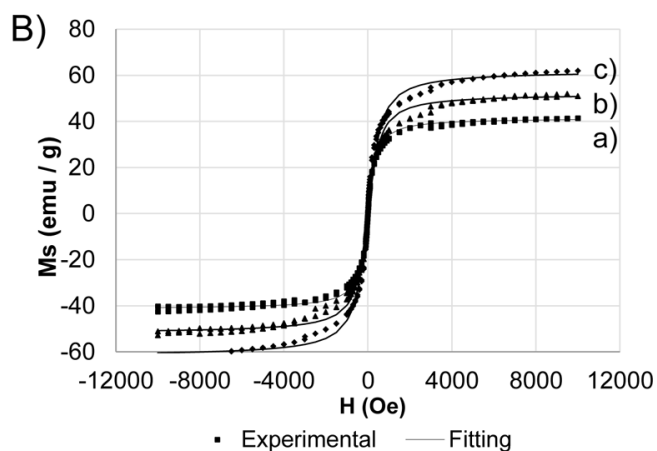

- Experimental _-Fitting

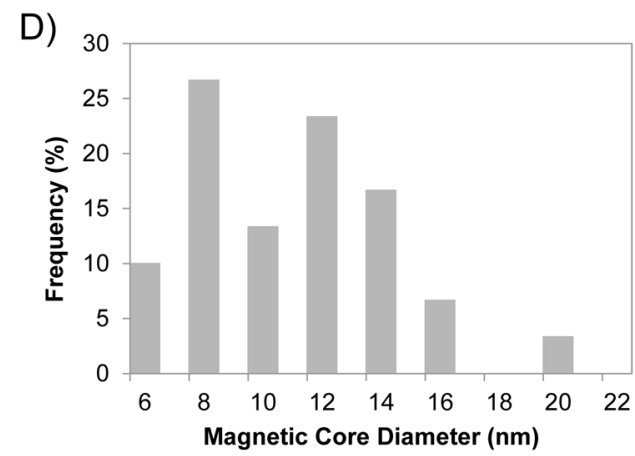

F)

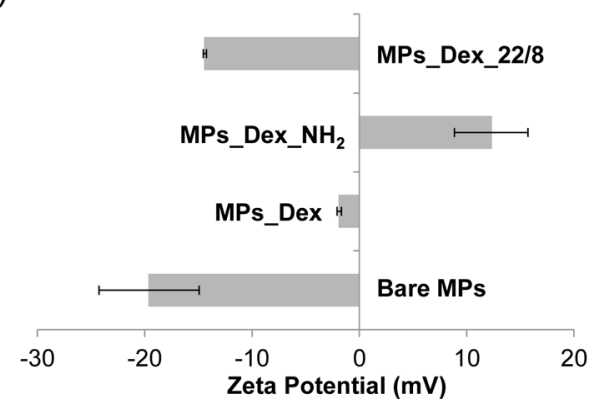

Figure 2. (A) Magnetic particle characterization by FTIR spectra for dextran (curve a), bare MPs (curve b), dextran-coated MPs (curve c), and MPs_Dex functionalized with 22/8 (curve d). (B) VSM curves for bare MPs (curve a), dextran-coated MPs (curve b), and MPs_Dex functionalized with $22 / 8$ (curve c). (C) TEM image of dextran-coated MPs. (D) Grain size distribution from TEM. (E) Hydrodynamic diameter. (F) Zeta potential $(n=2)$.

$235250 \mu \mathrm{L})$ in phosphate buffer $(50 \mathrm{mM}, \mathrm{pH} 8)$ were incubated with 250 $236 \mu \mathrm{L}$ at $6.1 \mathrm{mg} / \mathrm{mL}$ of MPs_Dex functionalized with ligand $22 / 8$ by 237 method C, as previously described in literature. ${ }^{21}$

238 Assessment of Monoclonal Antibody Magnetic Purification from 239 Crude Extracts. The functionalized (MPs_Dex_22/8 by Method C) 240 and nonfunctionalized supports (MPs_Dex $)(500 \mu \mathrm{L}$ with $54 \mathrm{mg} / \mathrm{mL})$ 241 were washed sequentially with regeneration and binding buffers, as 242 described above, and then incubated for $15 \mathrm{~min}$ at $4{ }^{\circ} \mathrm{C}$ with $500 \mu \mathrm{L}$ of 243 a $\mathrm{CHO}$ cell culture supernatant. The solution in which the particles 244 were suspended was removed by magnetic separation, and then MPs 245 were washed five times with binding buffer $(500 \mu \mathrm{L})$. After washing, 246 MPs were divided in two equal portions and protein recovery was 247 tested for two elution buffers: (i) $50 \mathrm{mM}$ glycine- $\mathrm{HCl}, \mathrm{pH} 3$ and (ii) $24850 \mathrm{mM}$ glycine- $\mathrm{NaOH}, \mathrm{pH}$ 11. All collected samples (loading, 249 flowthrough, and elutions) were analyzed by SDS-PAGE $12.5 \%$ 250 Acrylamide/Bisacrylamide in denaturing conditions and stained with 251 Silver Staining kit (BioRad). A BCA assay was also performed in order 252 to quantify the amount of total protein in each of the samples 253 collected.

\section{RESULTS AND DISCUSSION}

254 Preparation and Characterization of Affinity Magnetic 255 Supports. Magnetic supports were prepared by the chemical coprecipitation of iron salts and coated with dextran, a neutral 256 polysaccharide well-known as a coating agent. Upon MPs 257 coating, dextran presented high stability toward storage and 258 modification with amino-silanes, as no biopolymer was released 259 over a period of 160 days and during the amination step. The 260 prepared magnetic particles were then characterized by FTIR, 261 VSM, TEM and DLS. The analysis of FTIR spectra (Figure $2-262 \mathrm{fz}$ A) confirmed the presence of dextran on the surface of the 263 particles. The characteristic dextran peaks at $1427 \mathrm{~cm}^{-1}$, due to 264 $\mathrm{C}-\mathrm{H}$ bond bending, and around $1000 \mathrm{~cm}^{-1}$, due to the 265 stretching vibration of the alcoholic hydroxyl $(\mathrm{C}-\mathrm{OH})$, were 266 visible in the spectra of coated MPs. The characterization by 267 TEM revealed the existence of spherical magnetic cores (Figure 268 2C) with an average diameter of $12 \mathrm{~nm}$ (Figure 2D) and a size 269 distribution between $8-12 \mathrm{~nm}$, as observed previously by 270 Batalha and co-workers. ${ }^{21}$ The spherical magnetic cores tend to 271 form agglomerates, more pronounced upon dextran coating, as 272 assessed by an increase on the hydrodynamic diameter (Figure 273 $2 \mathrm{E}$ ) of MPs_Dex. This phenomenon has already been observed 274 in other works and might be attributed to the noncovalent 275 interactions between the coating biopolymers and neighbor 276 
A)

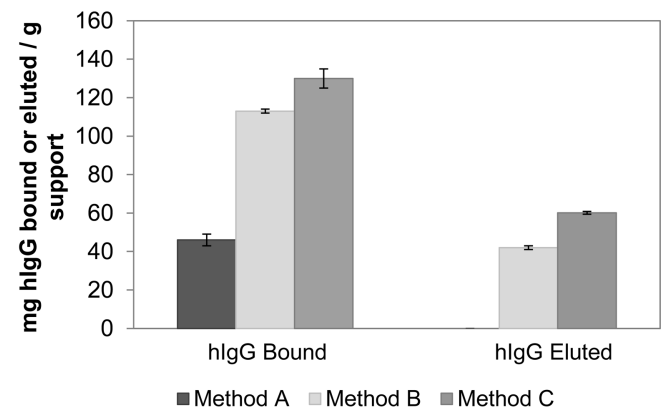

C)

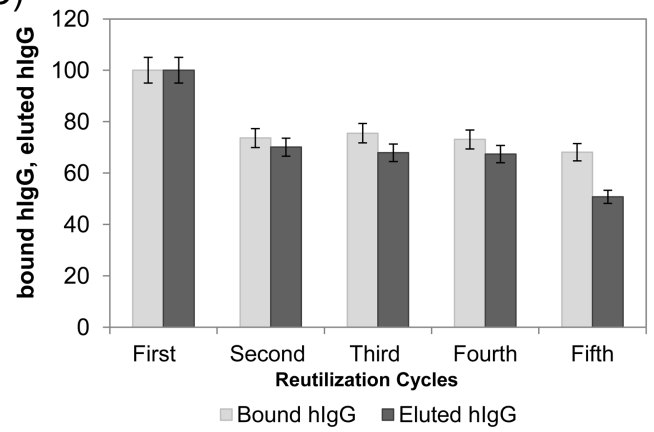

B)

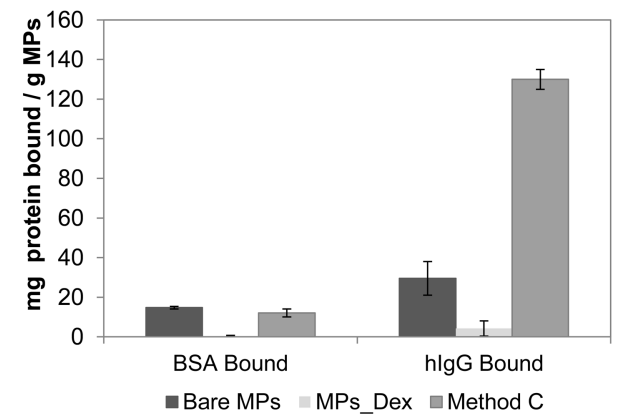

D)

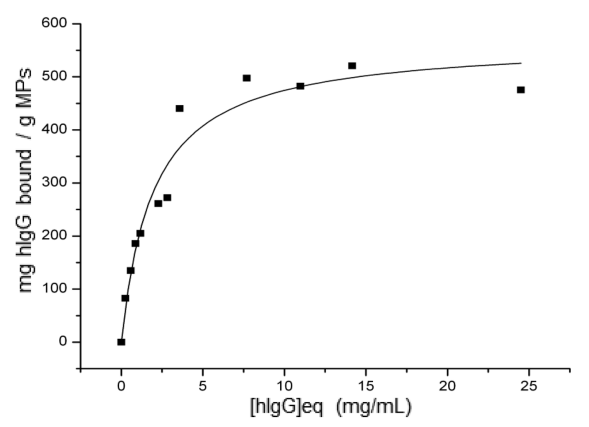

Figure 3. (A) Binding and elution of hIgG to MPs_Dex modified with ligand 22/8 $(n=2)$; (B) binding of BSA and hIgG to MPs_Dex modified with ligand 22/8 through Method C $(n=2)$; (C) reutilization of MPs_Dex modified with ligand 22/8 through Method C for binding and elution of hIgG $(n=2)$; and (D) binding of hIgG at the surface of MPs_Dex modified with ligand 22/8 by Method C. Representation of $q$ (the amount of bound hIgG in equilibrium per mass of solid support) as function of $C_{\mathrm{eq}}$ (the concentration of hIgG in equilibrium). Experimental data were fitted with the expression $q=\left(Q_{\mathrm{ma}} \times C_{\mathrm{eq}}\right) /\left(K_{\mathrm{d}}+\mathrm{C}_{\mathrm{eq}}\right)$ for the Langmuir isotherm (OriginLab 6.1 software), where $Q_{\max }$ corresponds to the maxium concentration of the matrix sites available to the partitioning solute (which can also be defined as the binding capacity of the adsorbent), and $K_{\mathrm{d}}$ is the dissociation constant $(n=2)$.

277 particles. $^{21,34}$ The hydrodynamic diameter for MPs_Dex 278 agglomerates decreases slightly upon modification with ligand $27922 / 8$, since this functionalization can create steric restrictions, 280 alteration of surface charge and increased hydrophobicity. ${ }^{21}$ 281 Through zeta potential analysis (Figure 2F), the presence of the 282 dextran was confirmed as well as the modification of the surface 283 of the particles with ligand $22 / 8$. When coated with dextran, the 284 particles presented a zeta potential of $-1.88 \mathrm{mV}$, because of the 285 neutral charge of the biopolymer, which is corroborated with 286 the values determine by $\mathrm{Xu}$ and co-workers. ${ }^{35}$ After chemical 287 modification of MPs_Dex with ligand 22/8, the zeta potential 288 of the supports became more negative. These changes in the 289 zeta potential show a surface charge rearrangement due to the 290 presence of new functionalization groups. ${ }^{21}$

291 Finally, through VSM analysis, it was possible to ascertain the 292 magnetic properties of the supports. The curves represented in 293 Figure 2B show reversibility and symmetry which represents a 294 typical no hysteresis curve characteristic of the super295 paramagnetic behavior of the particles synthesized. In terms 296 of saturation magnetization, the values obtained were 41.5 $297 \mathrm{emu} / \mathrm{g}$ for bare MPs (0.9955), $52.0 \mathrm{emu} / \mathrm{g}$ for MPs_Dex 298 (0.9946), and $62.0 \mathrm{emu} / \mathrm{g}$ for MPs_Dex modified with ligand $29922 / 8$ (0.9933). The saturation magnetization value obtained for 300 the bare MPs is consistent with the values referenced in the 301 literature. $^{36}$

302 Affinity Magnetic Separation of Antibodies. Our group 303 has previously shown the suitability of gum Arabic as a coating 304 agent to produce magnetic supports modified with the affinity 305 ligand $22 / 8$ for antibody separation. However, the charged 306 nature of gum Arabic can interfere with the adsorption of biocomponents and increase nonspecific interactions. The 307 inertness of MPs_Dex magnetic supports for binding hIgG 308 has been assessed and compared with bare agarose, the 309 traditional support for chromatography, bare MPs and gum 310 Arabic coated MPs. Agarose presented the lowest nonspecific 311 interactions $(0 \mathrm{mg} / \mathrm{g} \mathrm{hIgG}$ bound to unmodified agarose), 312 followed by MPs_Dex $(4 \pm 4 \mathrm{mg}$ of hIgG per gram of dried 313 MPs), MPs coated with gum arabic (28 $\pm 3 \mathrm{mg}$ of hIgG per 314 gram of dried MP), and bare MPs ( $60 \pm 2 \mathrm{mg}$ if hIgG per gram 315 of dried MP). ${ }^{21}$ MPs_Dex presented seven times less capacity 316 for binding to hIgG, when compared with gum Arabic coated 317 MPs. ${ }^{21}$ The differences in the chemical composition of the 318 biopolymers can explain the different reactivity they impair to 319 the magnetic supports. Nonetheless, coating MPs with 320 biopolymers is likely to create a net of porous structures that 321 leaves reactive iron oxide partly exposed to create interactions 322 and might have some contribution in the nonspecific 323 adsorption of each support.MPs_Dex particles were further 324 on explored for hIgG purification from pure solutions, through 325 the conjugation of a synthetic affinity ligand mimicking protein 326 A, named as ligand $22 / 8$. Three different methods for the 327 covalent attachment of the synthetic ligand onto MPs have 328 been tested (Figure 1). In method A, ligand $22 / 8$ was 329 synthesized in solution-phase with a six carbon spacer. In 330 method B, ligand 22/8 was also synthesized in solution-phase 331 but without a six carbon spacer. Finally, for method C, ligand 332 $22 / 8$ was synthesized directly on the solid support. In method 333 A, there is the need to use a strong cross-linker 334 (glutaraldehydem) which can also react with amine groups 335 from neighboring particles, therefore reducing the free aldehyde 336 
337 groups available to react with the amine groups from the ligand. 338 In addition, the solubility of the ligand is very poor. Method B 339 is performed at high temperature $\left(80-90^{\circ} \mathrm{C}\right)$, at which the less 340 reactive chloride of the ligand is substituted. Consequently, the 341 quantity of ligand that is immobilized on the support may be 342 compromised. In the case of method $\mathrm{C}$, this is a multistep 343 reaction where the coupling of the triazine ring is done at $0{ }^{\circ} \mathrm{C}$ 344 through the most reactive chloride, and therefore less likely to 345 result in low reaction yields. Previous works have also shown 346 that immobilization of very insoluble triazine ligands through 347 direct directly on the solid support yields best results for 348 protein adsorption. ${ }^{\text {? }}$

349 By analyzing the quantity of hIgG bound and eluted from the 350 supports (Figure $3 \mathrm{~A}$ ), method A revealed to be the less suitable 351 method followed by method B. Method C seems to be the best 352 method to immobilize ligand $22 / 8$ and to produce affinity 353 magnetic supports toward IgG To assess the recovery of 354 protein, we studied the elution buffer $50 \mathrm{mM}$ glycine- $\mathrm{NaOH}$, $355 \mathrm{pH} 11$, because of iron leaching at acidic $\mathrm{pH}$, previously 356 observed. ${ }^{21}$ In Method A it was not possible to quantify eluted 357 protein. In method B, it was possible to elute $42 \pm 1 \mathrm{mg}$ of $358 \mathrm{hIgG}$ eluted/g of MPs which corresponds to $37 \%$ of the bound 359 protein, whereas for method C, $46 \%$ of bound protein was 360 eluted. As a result of these studies, MPs_Dex with ligand 22/8 361 immobilized by Method C (MPs_Dex_228) appear as the 362 most promising magnetic supports with a binding capacity of $363130 \pm 5 \mathrm{mg}$ of $\mathrm{hIgG} / \mathrm{g}$ of MPs and a elution capacity of $60.1 \pm$ $3640.7 \mathrm{mg}$ of hIgG/g of MPs, and further studies were performed 365 with this magnetic support.

366 MPs_Dex_22/8 were tested for binding to a model 367 contaminant protein, bovine serum albumin (BSA), for which 368 the support should not present affinity. The magnetic support 369 bound $12 \pm 2 \mathrm{mg}$ of BSA/g of MP, a 10-fold lower value when 370 compared to the quantity of hIgG bound ( $130 \pm 5 \mathrm{mg}$ of hIgG $371 \mathrm{bound} / \mathrm{g}$ of $\mathrm{MP}$ ) (Figure $3 \mathrm{~B}$ ). The regeneration and reuse 372 capacity of the particles was also studied. As shown in Figure 373 3C particles retain about $70 \%$ of the initial protein binding and 374 elution capacity until the fifth stage of recycling. The $\mathrm{pH}$ 375 resistance of the support was evaluated in order to assess the 376 release of iron and dextran and therefore infer on eventual 377 ligand leaching, which is covalently bound to the polymer. The 378 total amount of dextran released after using five times the 379 support, was $0.0007 \%$ of the total amount of dextran initially 380 adsorbed, and during the first and second cycle of reutilization 381 there was no dextran release. In terms of magnetite release, we 382 observed that after five cycles of reutilization the support lost $3830.39 \%$ of the initial magnetite which corresponded to $19 \mathrm{ng}$ of 384 iron. In the first cycle of reutilization there was a leaching of $3850.09 \mathrm{mg} / \mathrm{L} \mathrm{Fe}$ (corresponding to $0.0006 \%$ of initial iron) during 386 the elution step, that is comparable with the results of Batalha 387 and co-workers. ${ }^{21}$ These observations, together with the 388 retention of protein attached to the support after elution and 389 regeneration, can account for the loss of capacity of the support 390 throughout the reutilization cycles.

391 The adsorption isotherm of human IgG on the magnetic 392 support MPs_Dex_22/8 (Figure 3D) was fitted in a Langmuir 393 type isotherm and compared with data available in the literature 394 (Table 1). The experimental adsorption values of human IgG 395 on MPs_Dex_22/8 was found to be $130 \mathrm{mg}$ of hIgG adsorbed/ $396 \mathrm{~g}$ of MPs. The commercially available protein A modified MPs 397 show experimental adsorption of $109 \mathrm{mg}$ hIgG adsorbed/g $398 \mathrm{MPs}^{37}$ Through the fitting of the adsorption curve of hIgG, an 399 affinity constant of $7.7 \times 10^{4} \mathrm{M}^{-1}\left(K_{\mathrm{a}}\right)$ and a theoretical
Table 1. Comparison of Binding Isotherm of Human IgG to Immobilized Protein A and Ligand 22/8 onto Different Supports and to Ligand 22/8 Immobilized on MPs_Dex through Method C

\begin{tabular}{lcc}
\multicolumn{1}{c}{ support } & $K^{\mathrm{a}}\left(\mathrm{M}^{-1}\right)$ & $\begin{array}{c}\mathrm{Q}_{\max }(\mathrm{mg} \text { of hIgG adsorbed/g of } \\
\text { nsupport })\end{array}$ \\
$\begin{array}{l}\text { protein A on agarose } \\
\text { commercial protein A on }\end{array}$ & $3.7 \times 10^{5}$ & 17 \\
$\quad$ MPs & $3.3 \times 10^{5}$ & 109 \\
ligand 22/8 on agarose & $1.4 \times 10^{5}$ & 152 \\
ligand 22/8 on cellulose & $3.0 \times 10^{5}$ & 630 \\
$\quad$ membrane & $1.5 \times 10^{5}$ & 344 \\
Ligand 22/8 on MPs_Ga & & 568 \\
ligand 22/8 on MPs_Dex & $7.7 \times 10^{5}$ & \\
\hline
\end{tabular}

maximum capacity of $568 \pm 33 \mathrm{mg}$ hIgG adsorbed/g MPs 400 $\left(Q_{\max }\right)$ were obtained with a correlation factor of 0.95 .The 401 affinity constant value is in the same order of magnitude to the 402 Protein A and ligand 22/8 immobilized on different supports. 403 The $Q_{\max }$ value for MPs_Dex_22/8 is nearly two times higher 404 than the same ligand immobilized on MPs_GA, ${ }^{21}$ four times 405 higher than the same ligand immobilized on agarose and thirty 406 times higher than the natural Protein A immobilized on 407 agarose..$^{7}$ Only the cellulose membrane revealed a higher 408 binding capacity, which was not compensated by the low 409 recovering capacity shown by this support. ${ }^{32}$

410

The magnetic support MPs Dex 22/8 was finally employed 411 in the small-scale purification of an IgG monoclonal antibody 412 directly from CHO cell culture supernatants (Figure 4 - A) $413 \mathrm{f} 4$ without any initial step to remove impurities. The recovery of 414 pure IgG was visible at $\mathrm{pH} 3$ and $\mathrm{pH}$ 11, but in larger yields for 415 the latter. From $56 \%$ of total protein bound to the support, 416 there was a recovery of 5 and $16 \%$ of total protein at $\mathrm{pH} 3$ and 417 11 , respectively (Figure 4C). Through analysis of the $2 \mathrm{D}$ gel by 418 densitometry analysis with software Image J, it was estimated 419 that the loading sample contains about $60 \%$ of IgG (in terms of 420 total protein present) and that the purified IgG presents $95 \% 421$ purity. The inertness of the MPs_Dex particles was also 422 assessed (Figure 4B) with the crude samples, showing the 423 absence of protein bound to or eluted from the support. 424

\section{CONCLUSION}

Iron oxide magnetic particles with a dextran coating are a 425 promising support for the magnetic separation of biomolecules, 426 because of the ease of preparation and chemical modification, 427 low cost, reduced nonspecific adsorption, and high stability. In 428 particular, the covalent attachment of a synthetic affinity ligand 429 mimicking protein A turned these particles viable for the one- 430 step recovery of IgG. Our results show that the direct synthesis 431 of the ligand on the magnetic support yielded the best 432 antibody-capturing properties. In addition, this support 433 MPs Dex 22/8 also showed low nonspecific adsorption in 434 the presence of BSA and no major loss of capacity after five 435 cycles of protein purification. Moreover the estimated values for 436 affinity constant for ligand $22 / 8$ were comparable with those 437 found for protein A and ligand 22/8 immobilized on different 438 adsorbents, but with the advantage of presenting considerable 439 higher maximum capacity for antibody adsorption. When 440 contacting the magnetic adsorbent with mammalian cell culture 441 supernatants rich in IgG, the MPs_Dex_22/8 supports were 442 able to purify IgG when eluting at $\overline{\mathrm{pH}} 11$ with a purity of $95 \% .443$ 

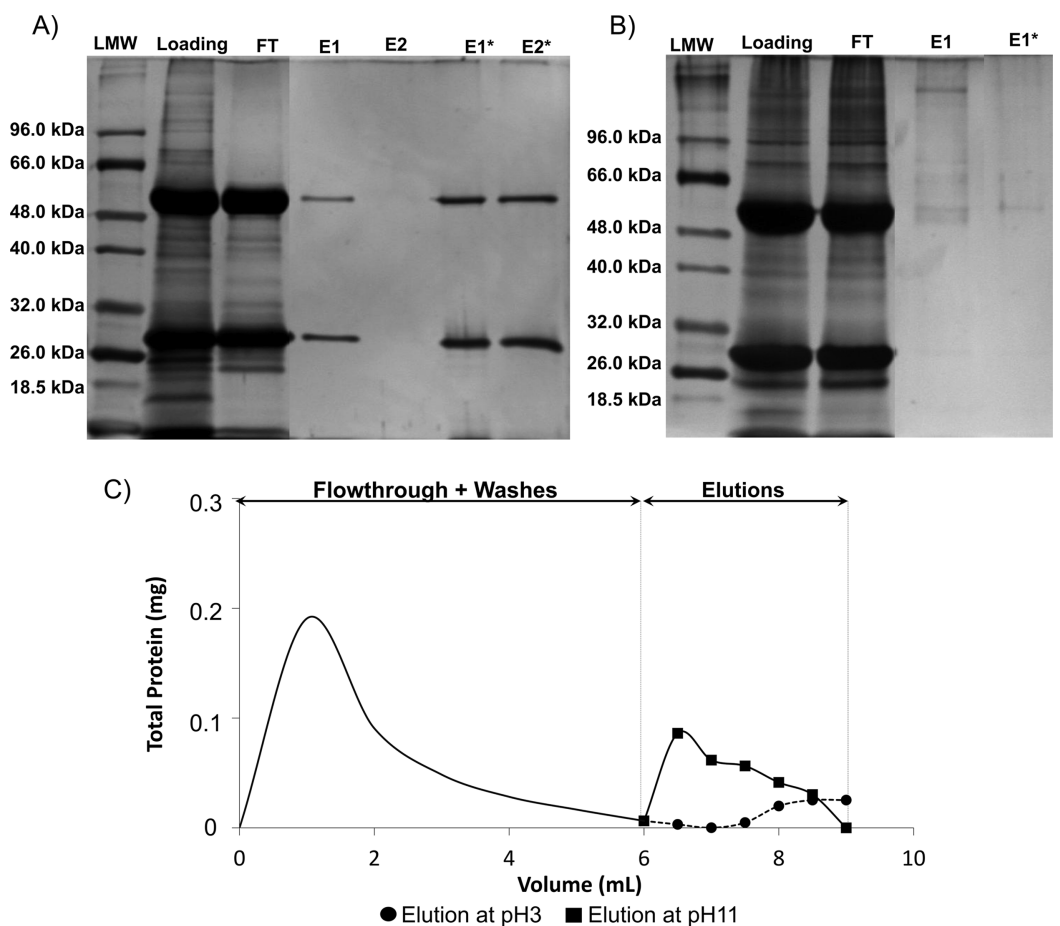

Figure 4. Electrophoreses gel $12.5 \%$ in denaturation conditions to verify (A) binding capacity of MPs_Dex_22/8 for IgG from a crude extract, (B) inertness of MPs_Dex for IgG. LMW (low molecular weight); loading (sample of the crude extract incubated with the adsorbent); FT (flowthrough); $\mathrm{E}^{-}$(first elution with $50 \mathrm{mM}$ glycine $-\mathrm{HCl}, \mathrm{pH} 3$ ); $\mathrm{E}^{*}$ (first elution with $50 \mathrm{mM}$ glycine $\left.-\mathrm{HCl}, \mathrm{pH} 11\right)$, and $(\mathrm{C})$ washes and elution profiles for IgG onto MPs_Dex_22/8. The squared and circled points represent the elution profiles at $\mathrm{pH} 11$ and 3, respectively.

\section{AUTHOR INFORMATION}

\section{Corresponding Author}

446 *E-mail: cecilia.roque@fct.unl.pt Tel.: +351212948385. Fax: $447+351212948550$.

\section{Notes}

449 The authors declare no competing financial interest.

\section{ACKNOWLEDGMENTS}

451 The authors thank the financial support from Fundação para a 452 Ciência e a Tecnologia through Grant PEst-C/EQB/LA0006/ 4532011 and contracts no. PTDC/EBB-BIO/102163/2008, 454 PTDC/EBB-BIO/098961/2008, PTDC/EBB-BIO/118317/ 455 2010, SFRH/BD/72650/2010 for V.L.D, and Santander 456 Totta Bank - Universidade Nova de Lisboa for the Scientific 457 Award 2009/2010. The authors are grateful to Dr. Abid 458 Hussain and M. Telma Barroso (REQUIMTE, FCT-UNL, 459 Portugal) for the preparation of the synthetic affinity ligands, to 460 Lonza Biologics, U.K. (Dr. Richard Alldread), and the Animal ${ }_{461}$ Cell Technology Unit of ITQB-UNL/IBET (Dr. Paula M Alves 462 and Dr. Ana Teixeira) for providing the cells and the culture 463 bulks and to Mr. Filipe Cardoso and Prof. Paulo Freitas 464 (INESC-MN, Lisbon, Portugal) for the help with the VSM 465 measurements.

466

\section{ABBREVIATIONS}

467 MPs, oxide magnetic particles; MPs_Dex, iron oxide magnetic 468 particles coated with dextran; MPs_GA, iron oxide magnetic 469 particles coated with gum Arabic; MPs_Dex_22/8, iron oxide 470 magnetic particles coated with dextran modified with ligand 22/ 471 8; hIgG, human IgG; BSA, bovine serum albumine

\section{REFERENCES}

(1) Wang, W.; Singh, S.; Zeng, D. L.; King, K.; Nema, S. J. Pharm. Sci. 473 2007, 96, 1 .

(2) Birch, J. R.; Racher, A. J. Adv. Drug Delivery Rev. 2006, 58, 671. 475

(3) Roque, A. C. A.; Silva, C. S. O.; Taipa, M.Â. J. Chromatogr., A 476 2007, 1160, 44.

(4) González, Y.; Ibarra, N.; Gómez, H.; González, M.; Dorta, L.; 478 Padilla, S.; Valdés, R. J. Chromatogr., B 2003, 784, 183.

(5) Kriangkum, J.; Xu, B.; Gervais, C.; Paquette, D.; Jacobs, F. A.; 480 Martin, L.; Suresh, M. R. Hybridoma 2000, 19, 33.

(6) Powers, D. B.; Amersdorfer, P.; Poul, M.-A.; Nielsen, U. B.; Shalaby, M. R.; Adams, G. P.; Weiner, L. M.; Marks, J. D. J. Immunol. 483 Methods 2001, 251, 123.

(7) Teng, S. F.; Sproule, K.; Husain, A.; Lowe, C. R. J. Chromatogr., 485 B: Biomed. Sci. Appl. 2000, 740, 1.

(8) Roque, A. C. A.; Taipa, M.Â.; Lowe, C. R. J. Mol. Recognit. 2005a, 487 18, 213.

488

(9) Roque, A. C. A.; Taipa, M.Â.; Lowe, C. R. J. Chromatogr., A 489 2005b, 1064, 157.

(10) Branco, R. J. F.; Dias, A. M. G. C.; Roque, A. C. A. J. 491 Chromatogr., A 2012, 1244, 106.

(11) Rosa, P. A. J.; Azevedo, A. M.; Sommerfeld, S.; Mutter, M.; 493 Aires-Barros, M. R.; Bäcker, W. J. Biotechnol. 2009, 139, 306.

(12) Ditsch, A.; Yin, J.; Laibinis, P. E.; Wang, D. I. C.; Hatton, T. A. 495 Biotechnol. Prog. 2006, 22, 1153.

(13) Laurent, S.; Forge, D.; Port, M.; Roch, A.; Robic, C.; Vander 497 Elst, L.; Muller, R. N. Chem. Rev. 2008, 108, 2064.

(14) Boyer, C.; Whittaker, M. R.; Bulmus, V.; Liu, J.; Davis, T. P. 499 NPG Asia Mater. 2010, 23.

(15) Dias, A. M. G. C.; Hussain, A.; Marcos, A. S.; Roque, A. C. A. Biotechnol. Adv. 2011, 29, 142.

(16) Lu, A.-H.; Salabas, E. L.; Schüth, F. Angew. Chem., Int. Ed. 2007, 503 46, 1222.

(17) Dawes, C. C.; Jewess, P. J.; Murray, D. A. Anal. Biochem. 2005, 505 338, 186. (1) .

(n)

\section{.}

\section{.}

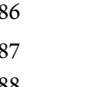


507 (18) Kievit, F. M.; Veiseh, O.; Bhattarai, N.; Fang, C.; Gunn, J. W.; 508 Lee, D.; Ellenbogen, R. G.; Olson, J. M.; Zhang, M. Adv. Funct. Mater. 509 2009, 19, 2244.

510 (19) Dung, T. T.; Danh, T. M.; Hoa, L. T. M.; Chien, D. M.; Duc, N. 511 H. J. Exp. Nanosci. 2009, 4, 259.

512 (20) Hradil, J.; Pisarev, A.; Babič, M.; Horák, D. China Particuol. $5132007,5$.

514 (21) Batalha, I. L.; Hussain, A.; Roque, A. C. A. J. Mol. Recognit. 2010, $51523,462$.

516 (22) Roque, A. C. A.; Bicho, A.; Batalha, I. L.; Cardoso, A. S.; 517 Hussain, A. J. Biotechnol. 2009, 144, 313.

518 (23) Kaittanis, C.; Nath, S.; Perez, J. M. PLoS ONE 2008, 3, e3253.

519 (24) Kaittanis, C.; Santra, S.; Perez, J. M. Adv. Drug Delivery Rev. 520 2010, 62, 408 .

521 (25) Heebøll-Nielsen, A.; Dalkiær, M.; Hubbuch, J. J.; Thomas, O. R. 522 T. Biotechnol. Bioeng. 2004a, 87, 311.

523 (26) Heebøll-Nielsen, A.; Justesen, S. F. L.; Hobley, T. J.; Thomas, 524 O. R. T. Sep. Sci. Technol. 2004b, 39, 2891.

525 (27) Huang, X.; Zhuang, J.; Chen, D.; Liu, H.; Tang, F.; Yan, X.; 526 Meng, X.; Zhang, L.; Ren, J. Langmuir 2009, 25, 11657.

527 (28) Diettrich, O.; Mills, K.; Johnson, A. W.; Hasilik, A.; Winchester, 528 B. G. FEBS Lett. 1998, 441, 369.

529 (29) Duan, H.; Shen, Z.; Wang, X.; Chao, F.; Li, J. World J. 530 Gastroenterol. 2005, 11, 3660.

531 (30) Massart, R. IEEE Trans. Magn. 1981, 17, 1247.

532 (31) Leyva, A.; Quintana, A.; Sánchez, M.; Rodríguez, E. N.; 533 Cremata, J.; Sánchez, J. C. Biologicals, 2008, 36,

534 (32) Barroso, T.; Temtem, M.; Hussain, A.; Aguiar-Ricardo, A.; 535 Roque, A. C. A. J. Membr. Sci. 2010, 348, 224.

536 (33) Sabaté, R.; Barnadas-Rodríguez, R.; Callejas-Fernández, J.; 537 Hidalgo-Álvarez, R.; Estelrich, J. Int. J. Pharm. 2008, 347, 156.

538 (34) Williams, D.; Gold, K.; Holoman, T.; Ehrman, S.; Wilson, O. J. 539 Nanopart. Res. 2006, 8, 749.

540 (35) Xu, X. Q.; Shen, H.; Xu, J. R.; Xu, J.; Li, X. J.; Xiong, X. M. Appl. 541 Surf. Sci. 2005, 252, 494.

542 (36) Gupta, A. K.; Wells, S. IEEE Trans. Nanobiosci. 2004, 3, 66.

543 (37) Borlido, L.; Azevedo, A. M.; Roque, A. C. A.; Aires-Barros, M. R.

544 J. Chromatogr., A 2011, 1218, 7821. 